\title{
Marketing, Gestión de la Calidad Total y Benchmarking: una revisión de la literatura
}

\section{Marketing, Total Quality Management and Benchmarking: a review of the literature}

\author{
Sherly Hoyos-Estrada \\ Universidad Tecnológica de Bolívar - Colombia \\ ORCID iD: https://orcid.org/0000-0003-2533-5344 \\ shhoyos@utb.edu.co
}

\author{
Fecha de recepción: 06/08/2021 \\ Fecha de evaluación: 24/08/2021 \\ Fecha de aceptación: 30/09/2021 \\ Cómo citar: Hoyos-Estrada, S. (2021). Marketing, Ges-
tión de la Calidad Total y Benchmarking: una revi-
sión de la literatura. Revista Cientifica Anfibios, 4(2),
64-71.
https://doi.org/10.37979/afb.2021v4n2.96
}

\section{Resumen}

El marketing ocupa uno de los lugares centrales al interior de las estrategias de las organizaciones en la actualidad debido a lo inestable del entorno empresarial, es por esto que su gestión y los elementos inherentes a ella son de vital importancia para el entorno organizacional actual. En este contexto, se busca investigar los vínculos entre el marketing y la gestión de la calidad total (TQM), por medio de una revisión de literatura que analice diversas publicaciones en torno a esta temática, en donde se encuentran similitudes y afinidades en los hallazgos entre estas dos áreas, sin embargo, en el mundo real y en la práctica hay una aparente falta de integración de las dos. Por tanto, se realizó un estudio que muestra que los académicos de marketing están menos dispuestos a utilizar métodos de benchmarking que otros grupos y aún es menos probable que los gerentes la estén utilizando. Es así que los principales hallazgos se centran en explorar como la integración entre la TQM, el Benchmarking y el marketing puede favorecer el entorno empresarial de las organizaciones.

Palabras clave

Marketing; TQM; benchmarking; estrategia

\begin{abstract}
Marketing occupies one of the central places within the strategies of organizations today due to the unstable business environment, which is why its management and the elements inherent to it are of vital importance for the current organizational environment. In this context, we seek to investigate the links between marketing and total quality management (TQM), through a literature review that analyzes various publications on this topic, where similarities and affinities are found in the findings between these two areas, however, in the real world and in practice there is an apparent lack of integration of the two. Therefore, a study was conducted showing that marketing academics are less willing to use benchmarking methods than other groups and managers are even less likely to be using it. Thus, the main findings focus on exploring how integration between TQM, benchmarking and marketing can benefit the business environment of organizations.
\end{abstract}


Keywords

Marketing; TQM; benchmarking; strategy

\section{Introducción}

El marketing es un pilar fundamental dentro de cualquier empresa que desee implementar un sistema de gestión de calidad (Longbottom et al., 2000) dado que esto implica involucrarse directamente con el cliente y la responsabilidad que esto conlleva, ya que en un entorno empresarial actual, el cliente cada vez hace más conciencia de la calidad que se exige en el bien o servicio esperado a recibir, el uso de normas ISO asociadas al sector de calidad es fundamental para poder responder acorde a dicha área, haciendo cada día un mercado más exigente en este tema(Prayudha \& Harsanto, 2020; Saleh \& Watson, 2017).

Existe una relación difusa entre lo que es calidad de producto y servicio, satisfacción del cliente y la rentabilidad en su conjunto con aquello que esto significa para cada organización (Saleh \& Watson, 2017). Los clientes exigen alta calidad en los productos y servicios, aunque esto se vea reflejado en el precio final de su adquisición, mientras las empresas piensan en todo momento en como bajar los costos de producción para ser más redituable (Gillen, 2008). Tomando otra teoría a la definición de calidad entendida como la totalidad de características de un producto o servicio que tienen que ver con su capacidad para satisfacer las necesidades implícitas declaradas

Para Nazar et al., (2018) la definición técnica de gestión de calidad hace referencia a una estrategia de gestión empresarial que consiste en el estudio y valoración del concepto de calidad en cada una de las fases de un proceso de producción. La finalidad es la mejora constante de bienes y servicios ofertados y la consecución de mayor satisfacción del cliente.

Otra forma de comprender este concepto a través de su traducción al idioma ingles TQM (Total Quality Management). Estas nomenclaturas son totalmente comprendidas desde la calidad que es exigida y evaluada a través de recolección de distintos elementos que un grupo recolecta dentro de una empresa (R. Kumar \& Mishra, 2020). Es decir, siempre prevalece la continua búsqueda de la calidad en distintos procesos de la organización. Este concepto nació en los años 50 gracias a la nación japonesa que de la mano del sector industrial llevo a un mayor reconocimiento en 1983 en occidente gracias a un estudio publicado en la Harvard Business Review (Aich et al., 2018; Longbottom et al., 2000).

Los autores estudian los vínculos entre el marketing y gestión de calidad total y en particular la función del benchmarking, aunque en la búsqueda literaria se encontraron similitudes entre las definiciones y procesos de TQM y benchmarking, aún faltan muchos estudios que muestren los beneficios entre los dos métodos integrados (Hansson \& Eriksson, 2002).

El articulo intenta demostrar que existe una relación fuerte entre la orientación de la mercadotecnia y TQM. Se encontró en la literatura acerca de TQM, que existen dos paradigmas en este punto de vista, los cuales describen TQM como un modelo económico. Argumentan que existe varias diferencias y que son evidentes a través de un rango de factores críticos cuando se compara TQM con un modelo económico. Es por esto que se parten de unos referentes teóricos en donde se muestren los principales conceptos asociados a la temática en estudio. Seguidamente se presentan los aspectos metodológicos tomando como referencia una metodología basada en una revisión de la literatura y presentar los resultados de dichos análisis. Finalmente se proponen algunas conclusiones y futuras investigaciones.

\section{Referentes Teóricos}

La orientación del marketing tiene que ver con el logro de los objetivos corporativos mediante el cumplimiento de superar las expectativas de los clientes mejor que la competencia y esto se logra a través del estudio de las necesidades de los clientes y el trabajo integrado con esfuerzos enfocados al cliente (Sreedharan et al., 2018; Jobber, 1998).

Específicamente el benchmarking es una herramienta válida para la autoevaluación, para la evaluación comparativa de rendimiento de la empresa y para la búsqueda de mejores prácticas de empresas (Singh et al., 2018) 
Por consiguiente, la gestión de la calidad total es entendido por Iqbal \& Asrar-ul-Haq, (2018) como un sistema de gestión orientado a las personas tiene como objetivo mejorar continuamente la satisfacción del cliente con costos reales más bajos, es decir, es un enfoque total y una parte integral de la estrategia de alto nivel.

En la revisión de literatura hay evidencia significativa de que la implementación de la gestión total de calidad y modelos de excelencia organizacional desarrollan una mejoría en el desempeño financiero (Kumar, V., \& Sharma; 2017; Sohal \& Terziovski 2000;Longbottom et al., 2000). Sin embargo, la implementación es compleja y de largo plazo.

Para Najm et al., (2017) existe una diferencia entra la orientación entre TQM, benchmarking y el modelo económico; la orientación de TQM y marketing van encaminados a la satisfacción del cliente, mientras que el modelo económico va orientado a la maximización de ganancias minimizando el esfuerzo, la estructura organizacional de TQM Y marketing va más enfocada al coaching, funciones cruzadas, proceso y equipos, adaptación el modelo económico se enfoca más en la teoría $\mathrm{x}$ (poder $\mathrm{y}$ autoridad)(Grant et al., 1995). De aquí la importancia en la investigación de los vínculos entre TQM y marketing.

En este contexto, existe poco trabajo empírico que vincula la mercadotecnia con TQM generalmente o con benchmarking en particular, aunque existen varios artículos conceptuales identificando la relación positive entre estos elementos. Existen similaridades entre métodos benchmarking (puntos de referencia) y el servicio de blueprinting. Actualmente se intenta utilizar puntos de referencia en la planeación de la mercadotecnia, con el fin de evitar la Perdida en del enfoque y caer en procesos obsoletos. Se ha encontrado evidencia considerable de un movimiento hacia la adopción de estas ideas y vínculos positivos con la mejora del desempeño, aunque también se identificó que la implementación es difícil y a largo plazo.

Las organizaciones tienen a seguir un solo estilo y esto va a influenciar su comportamiento y la manera en que toman decisiones. Es posible observar los distintos factores en la tabla 1.

Tabla 1. Paradigmas de gestión emergentes: TQM y el modelo económico de la empresa

\begin{tabular}{|c|c|c|c|}
\hline & TQM & Modelo económico & Orientación al marketing \\
\hline Objetivos & $\begin{array}{l}\text { Satisfacción del } \\
\text { cliente }\end{array}$ & $\begin{array}{l}\text { Maximización de los } \\
\text { beneficios }\end{array}$ & $\begin{array}{l}\text { Creación de valor y beneficios a través } \\
\text { de la satisfacción del cliente }\end{array}$ \\
\hline Motivación & $\begin{array}{l}\text { Realización } \\
\text { personal y } \\
\text { aceptación social }\end{array}$ & $\begin{array}{l}\text { Maximizar los } \\
\text { ingresos y minimizar } \\
\text { el esfuerzo }\end{array}$ & $\begin{array}{l}\text { Cultura de valores y creencias } \\
\text { compartidas y enfoque de toda la } \\
\text { organización en el cliente }\end{array}$ \\
\hline Orientación & $\begin{array}{l}\text { Dinámica: } \\
\text { innovación y } \\
\text { mejora continua }\end{array}$ & $\begin{array}{l}\text { Estático: maximizar } \\
\text { los flujos de caja, los } \\
\text { ingresos y el coste }\end{array}$ & $\begin{array}{c}\text { Acoger el cambio, invertir en innovación } \\
\text { y ser rápidos }\end{array}$ \\
\hline Estilo de gestión & Teoría y: coaching & $\begin{array}{l}\text { Teoría } \mathrm{x} \text { : poder y } \\
\text { autoridad }\end{array}$ & Adaptación al entorno y a la complejidad \\
\hline $\begin{array}{l}\text { Estructura de la } \\
\text { organización }\end{array}$ & Interfuncional & $\begin{array}{l}\text { Funcional y } \\
\text { jerárquico }\end{array}$ & $\begin{array}{c}\text { Coordinación interfuncional y adaptación } \\
\text { a entornos complejos }\end{array}$ \\
\hline Sistema de información & $\begin{array}{l}\text { Acceso abierto, } \\
\text { aprendizaje y } \\
\text { desarrollo }\end{array}$ & $\begin{array}{l}\text { Acceso restringido, } \\
\text { control y formalidad }\end{array}$ & $\begin{array}{l}\text { Orientación a la investigación, flujos de } \\
\text { información y comunicación vitales }\end{array}$ \\
\hline Ventaja competitiva & Mejora continua & Ventaja comparativa & $\begin{array}{l}\text { Mejora continua y competencias } \\
\text { distintivas que proporcionen un valor } \\
\text { mejor que el de los competidores }\end{array}$ \\
\hline $\begin{array}{l}\text { Relaciones con } \\
\text { los proveedores/ } \\
\text { distribuidores }\end{array}$ & Relacional & Transaccional & Relacionales y en red \\
\hline Orientación al cliente & Relacional & Transaccional & Cada vez más relacional \\
\hline Enfoque estratégico & Cliente & Competidor & Cliente y competidor \\
\hline
\end{tabular}

Fuente: Longbottom et al., (2000). 
Estos estilos dependen en gran medida de las tipologías de benchmarking que maneje la organización, es por esto que se mencionan las 3 principales destacando que existen muchas más.

\section{Blueprinting}

La necesidad de innovación en esta economía mundial y el enfoque de muchos negocios de crear valor a través de mejorar la experiencia del cliente sugiere una necesidad de innovar métodos, técnicas y prácticas de investigación y Desarrollo para servicios (Buics, 2020). Service Blueprinting tiene un enfoque basado en el cliente para la innovación y mejora del servicio. Mientras que los fundamentos de service blueprinting fueron presentadas hace dos décadas, el método ha evolucionado significativa usando un acercamiento para afrontar los diversos retos en el diseño de servicios y su innovación (Istanbouli, 2021). Una de las características más distintivas de los servicios es su naturaleza.

A diferencia de los bienes tangibles, los servicios son dinámicos, se desarrollan a través de un periodo de tiempo a través de una secuencia de eventos y pasos. El proceso de servicio puede ser visto como una cadena de actividades que permite que el servicio funcione efectivamente. Service Blueprinting en rasgos generales es un diagrama de flujo donde se representa de manera visual los pasos o actividades de un negocio (Bitner et al., 2008).

El benchmarking competitivo busca medir los productos, servicios, procesos y funciones de los principales competidores para realizar una comparación con nuestra empresa y poder detectar y llevar a cabo mejoras que superen a las de nuestros competidores (Javaid et al., 2017).

Quizás sea el más complicado de llevar a cabo de los tres tipos, puesto que el análisis y el estudio como ya he mencionado se realizan sobre los principales competidores. Al considerarse tu competencia directa, en la gran mayoría de los casos no están interesados en colaborar. ¿Esto quiere decir que si no colaboran no lo podamos llevar a cabo? Por supuesto que no, pero obviamente en la recopilación de los datos necesarios se deberán emplear más recursos, y por tanto será mucho más costosa.

\section{Internal Benchmarking}

De acuerdo con Piran et al., (2021) El benchmarking interno se lleva a en la misma empresa. Suele realizarse en una gran empresa con diferentes departamentos o en un grupo empresarial compuesto por varias empresas. En este proceso, identificar un departamento o área que, por sus buenos resultados, pueda ser emulado para el benchmarking con otros departamentos internos de la empresa.

Es el más fácil de realizar dentro de compañías con estructuras con un cierto tamaño, además normalmente es el que menos recursos necesita para llevarlo a cabo, ya que la información se obtiene de la propia empresa.

\section{Funcional Benchmarking}

El benchmarking funcional permite identificar las mejores prácticas de las empresas que se desempeñan bien en las áreas a mejorar. Esta empresa no es necesariamente un competidor o incluso pertenece a la misma industria. (Dadi et al., 2019).

Normalmente es muy productivo, dado que al no tratarse de organizaciones que no son competidoras directas no existe un problema de confidencialidad y se suele ofrecer la información necesaria para el estudio.

\section{Metodología}

Basado en un estudio de corte cualitativo y en específico de corte analítico-descriptivo en el cual se plantea examinar la relación entre el Marketing, Gestión De La Calidad Total y Evaluación Comparativa. Para tales fines se plantea una revisión sistemática de la literatura (Tranfield et al., 2003), en la cual se evidencien los aportes mas significativos vinculando estas temáticas. Estas publicaciones se tomaron de Bases de datos de científicas como Scopus y Web Of Science (WOS) con la finalidad de obtener los resultados con mayor impacto en el ámbito investigativo. Posteriormente se seleccionaron las publicaciones directamente relacionadas con el Marketing, Gestión De La Calidad Total Y Evaluación Comparativa por medio de filtros y palabras claves que posibilitaron obtener las 
publicaciones con mayor rigor académico. Finalmente se analizaron comparativamente y se expusieron los principales resultados.

\section{Resultados}

Como resultado de la revisión de la literatura Okland (2000) afirmó que la calidad comienza con el marketing, mientras que Hooley (1993) y Kotler (1998) creen que la calidad debe estar dirigida por el mercado. Sin embargo, los autores de este artículo encuentran que en la práctica existe una falta de integración de técnicas, descubrieron que el benchmarking es un método de investigación apropiado para las disciplinas de TQM como de marketing, por lo tanto, abordan el trabajo de investigación para explorar sus similitudes y diferencias a nivel conceptual pero también a profundidad mediante un cuestionario dirigido a gerente y ejecutivos.

Se observa la importancia que le dan los académicos del TQM y el sector manufacturero al benchmarking mientras que otros sectores como el de servicios consideran el benchmarking como rutina.

La investigación confirmó la sugerencia de que existe una falta de integración de técnicas entre TQM y marketing ya que cuando se les pidió a los gerentes de marketing que califican el valor de una técnica de TQM solo por su nombre, estos otorgaron calificaciones bajas y poca relevancia a sus actividades, por el contrario cuando las técnicas se presentaron en un formato diferente, sin el nombre pero con una definición, explicación y ejemplo de uso, los encuestados identificaron un alto nivel de importancia para las actividades de marketing, esto lleva a concluir que hay un margen considerable para un mejor desarrollo de técnicas a fin de hacerlas más atractivas.

Toda empresa que pone en acción estrategias de calidad, no debería limitarse únicamente a la repercusión económica que este conlleva para la empresa, ya que este beneficio monetario no es una meta unitaria, sino, hablar de calidad conlleva el satisfacer las necesidades esperadas por el consumidor final, desarrollando una imagen corporativa de cultura de responsabilidad social. La implementación del marketing de una empresa no solo debe realizarse de forma externa, sino también interna generando un marketing orientado.
La implementación de Benchmarking dentro de una empresa es con el simple propósito que surge como opción ante un inminente acceso de información que genera la calidad en bienes o servicios de otra empresa. Esto no significa que no es copiar o robar ideas de la competencia, sino, todo lo contrario, es aprender de ellas con el fin de observar y mejorar áreas de oportunidad dentro de nuestra empresa, así nos relata Hernández y Cano (2017). Por lo antes mencionado, compararse con las mejores organizaciones es tener la oportunidad de crecer, mejorar procesos, satisfacer las expectativas del cliente y finalmente fortalecer la calidad en el bien o servicio, retomando la misma idea dentro del artículo de Hernández \& Cano (2017). La importancia del Benchmarking como herramienta para incrementar la calidad en el servicio en las organizaciones, nos relatan que para estudiar el Benchmarking nos podemos situar en dos épocas, La primera haciendo referencia a un concepto de herramienta que mejore la competitividad de la empresa haciéndola diferente a las demás, tratándose de un proceso continuo para ser mejorando las prácticas de la empresa.

Camp nos define este concepto como "La búsqueda de las prácticas de la industria que conducen a un desempeño excelente" (Camp, 1993).

En la 2da época, el Benchmarking nos plantea estudiar como una empresa ha podido establecer una línea de liderazgo dentro de su sector. Muchos autores insisten que la base del Benchmarking no es adquirir u observar las mejores prácticas de la competencia, sino, comprender como hacen las cosas de manera eficaz y eficiente. (Camp, 1993). Para Coldling ( 2000 citado por Hernández y Cano 2017), el Benchmarking se está convirtiendo en una forma eficaz de transmitir el conocimiento y las vivencias de otras empresas para la generación de nuevas y mejores ideas. El proceso no termina solo con aprender de otras organizaciones sino con crear planes estratégicos para ser implementados dentro de las propias empresas.

\section{Conclusión}

Es evidente las similitudes que existen entre las actividades y enfoque de la Gestión de Calidad Total y benchmarking, enfocados al cumplimiento de objetivos y la mejora continua, aunque el TQM lo hace desde un sistema de gestión y el benchmarking por medio de la evaluación comparativa. 
El benchmarking practica un ejercicio de búsqueda, comparación y aprendizaje de las mejores prácticas que está realizando la competencia, su objetivo es implementar las mejores estrategias para operar de forma eficiente y mantenerse como el mejor, la Gestión de Calidad Total también, pero esta lleva un proceso más complejo y más largo.

Es importante que los planificadores estratégicos y los gerentes diseñan planes corporativos, planes estratégicos de marketing y de gestión de calidad de manera que los esfuerzos de la mejora del desempeño interno de la organización no se desperdicien, sino que busquen oportunidades externas en las que la organización se pueda enfocar (Longbottom, Mayer y Casey, 2000).

En lo personal considero que las dos prácticas son igual de importantes y útiles, lo recomendable sería vincularlas de manera estratégica.
En esta parte del trabajo podría nuevamente exactamente repetir la conclusión del articulo número dos de la semana pasada, donde nos podemos poner a reflexionar que, nuevamente como dice Denison y McDonald (1995):

"El éxito en marketing no consiste solamente en identificar cuál es la estrategia adecuada, sino, además, en poder implantarla correctamente".

Ya se mencionaba en escritos anteriores que el Benchmarking no solo era el tomar las mejore prácticas de nuestra competencia sino implementarlas, mucho análisis provoca parálisis, jasí que acción! Esa sería la palabra de este artículo, llevar a la acción todas estas teorías dadas, "Comprender el concepto marketing es una cosa, ponerlo en práctica es otra" Lambin (1995).

\section{Referencias}

Africano, N., Rodrigues, A. S., \& Santos, G. (2019). The Main Benefits of the Implementation of the Quality Management System in Higher Education Institutions in Angola. Quality Innovation Prosperity, 23(3), 122-136. https://doi.org/10.12776/qip.v23i3.1292

Aich, S., Muduli, K., Onik, M. M. H., \& Kim, H. C. (2018). A novel approach to identify the best practices of quality management in SMES based on critical success factors using interpretive structural modeling (ISM). International Journal of Engineering and Technology(UAE), 7(3), 130-133. https://doi.org/10.14419/ijet.v7i3.29.18540

Bitner, M. J., Ostrom, A. L., \& Morgan, F. N. (2008). Service blueprinting: A practical technique for service innovation. California Management Review, 50(3), 66-94. https://doi. org/10.2307/41166446

Bravi, L., Murmura, F., \& Santos, G. (2019). The ISO 9001:2015 Quality Management System Standard: Companies' Drivers, Benefits and Barriers to Its Implementation. Quality Innovation Prosperity, 23(2), 64-82. https://doi.org/10.12776/qip.v23i2.1277

Buics, L. (2020). Analyzing Public Service Processes from Customer and Employee Perspectives by Using Service Blueprinting and Business Process Modelling. September, 195-211.

Dadi, K., Rahim, M., Abraham, A., Chyzhyk, D., Milham, M., Thirion, B., \& Varoquaux, G. (2019). Benchmarking functional connectome-based predictive models for resting-state fMRI. NeuroImage, 192(February), 115-134. https://doi.org/10.1016/j.neuroimage.2019.02.062

Gillen, D. (2008). Benchmarking and Performance Measurement: The Role in Quality Management. 325-338. https://doi.org/10.1108/9780080435930-020

Grant, R. M., Shani, R., \& Krishnan, R. (1995). TQM's challenge to management theory and practice. MIT Sloan Management Review, 35(2).

Gurutze, I., \& Velasco, E. (2010). El benchmarking aplicado a la gestión de la innovación. Revista de Dirección y Administración de Empresas. 
Hansson, J., \& Eriksson, H. (2002). The impact of TQM on financial performance. Measuring Business Excellence, 6(4), 44-54. https://doi.org/10.1108/13683040210451714

Hernández, C. \& Cano, M. (2017). La importancia del Benchmarking como herramienta para incrementar la calidad en el servicio en las https:/economipedia.com/definiciones/ gestion-la-calidad-total.html

Iqbal, A., \& Asrar-ul-Haq, M. (2018). Establishing relationship between TQM practices and employee performance: The mediating role of change readiness. International Journal of Production Economics, 203(July 2017), 62-68. https://doi.org/10.1016/j.ijpe.2018.05.034

Istanbouli, B. (2021). Blueprinting of a Normalized Supply Chain Processes : Results in Implementing Normalized Software Systems. January.

Javaid, A., Kurjakovic, S., Masuda, H., \& Kohda, Y. (2017). Enabling digital transformation in SMEs by combining enterprise ontologies and service blueprinting. Lecture Notes in Computer Science (Including Subseries Lecture Notes in Artificial Intelligence and Lecture Notes in Bioinformatics), 10371 LNCS, 224-233. https://doi.org/10.1007/978-3-319-61240-9_21

Kumar, R., \& Mishra, R. S. (2020). Linking TQM Critical Success Factors to strategic goal : Impact on Organizational Performance. IOSR Journal of Mechanical and Civil Engineering, 17(3), 1-13. https://doi.org/10.9790/1684-1703020113

Kumar, V., \& RRK, S. (2017). An empirical investigation of critical success factors influencing the successful TQM implementation for firms with different strategic orientation. International Journal of Quality \& Reliability Management, 34(9), 1-5.

Longbottom, D., Mayer, R., \& Casey, J. (2000). Marketing, total quality management and benchmarking: Exploring the divide. Journal of Strategic Marketing, 8(4), 327-340. https://doi. org/10.1080/096525400446221

Magd, H., Negi, S., \& Ansari, M. S. A. (2021). Effective TQM Implementation in the Service Industry: A Proposed Framework. Quality Innovation Prosperity, 25(2), 95-129. https://doi. org/10.12776/qip.v25i2.1594

Najm, N. A., Yousif, A. S. H., \& Al-Ensour, J. A. (2017). Total quality management (TQM), organizational characteristics and competitive advantage. Journal of Economic \& Financial Studies, 5(04), 12. https://doi.org/10.18533/jefs.v5i04.293

Nazar, N., Ramzani, S. R., Anjum, T., \& Shahzad, I. A. (2018). Organizational Performance: The Role of TQM Practices in Banking Sector of Pakistan. European Scientific Journal, ESJ, 14(31), 278. https://doi.org/10.19044/esj.2018.v14n31p278

Piran, F. S., Lacerda, D. P., Camanho, A. S., \& Silva, M. C. A. (2021). Internal benchmarking to assess the cost efficiency of a broiler production system combining data envelopment analysis and throughput accounting. International Journal of Production Economics, 238(November 2020), 108173. https://doi.org/10.1016/j.ijpe.2021.108173

Prayudha, A. N., \& Harsanto, B. (2020). Integration of service quality, benchmarking and Ishikawa diagram in service operations. Jurnal Manajemen Dan Pemasaran Jasa, 13(2), 151. https://doi. org/10.25105/jmpj.v13i2.6144

Saleh, A., \& Watson, R. (2017). Business excellence in a volatile, uncertain, complex and ambiguous environment (BEVUCA). The TQM Journal, 29(5), 705-724. https://doi.org/10.1108/tqm-122016-0109

Sánchez, J. (2017). Economipedia; “Gestión de calidad total”. 
Singh, V., Kumar, A., \& Singh, T. (2018). Impact of TQM on organisational performance: The case of Indian manufacturing and service industry. Operations Research Perspectives, 5, 199-217. https://doi.org/10.1016/j.orp.2018.07.004

Sohal, A. S., \& Terziovski, M. (2000). TQM in Australian manufacturing: Factors critical to success. International Journal of Quality and Reliability Management, 17(2), 158-167. https://doi. org/10.1108/02656710010304564

Sreedharan, R. V., Sunder, V. M., \& Raju, R. (2018). Critical success factors of TQM, Six Sigma, Lean and Lean Six Sigma: A literature review and key findings. Benchmarking, 25(9), 34793504. https://doi.org/10.1108/BIJ-08-2017-0223

Tošenovský, J., Tošenovský, F., \& Dočkalová , I. . (2021). Effects of Systematic Changes in Commodity Arrangement on the Satisfaction of Supermarket Customers. Quality Innovation Prosperity, 25(1), 16-32. https://doi.org/10.12776/qip.v25i1.1497

Tranfield, D., Denyer, D., \& Smart, P. (2003). Towards a Methodology for Developing Evidence-Informed Management Knowledge by Means of Systematic Review* Introduction: the need for an evidence- informed approach. British Journal of Management, 14, 207-222. 